\title{
FATORES PROGNÓSTICOS DE MORTALIDADE EM PACIENTES COM TRAUMA CARDÍACO QUE CHEGAM À SALA DE CIRURGIA
}

\author{
PREDICTORS OF MORTALITY IN PATIENTS WITH CARDIAC TRAUMA WHO \\ ARRIVE ALIVE IN THE OPERATION ROOM
}

\author{
Fernando Antonio Campelo Spencer Netto, TCBC-PE ${ }^{1}$ \\ Josemberg Marins Campos, ACBC-PE ${ }^{1}$ \\ Luís Filipe Cavalcanti Lima, ACBC-PE ${ }^{1}$ \\ Marco Antonio C. P. Rivera ${ }^{2}$ \\ Flávio Kreimer ${ }^{2}$ \\ Raquel Kelner Silveira, ACBC-PE ${ }^{3}$
}

\begin{abstract}
RESUMO: Objetivo: Detectar índices de complicações pós-operatórias e de letalidade, identificando fatores de risco (mecanismo de trauma, sítio da lesão cardíaca, local da intervenção cirúrgica, lesões associadas, necessidade de transfusão e escores anatômicos de trauma) em pacientes com traumatismo cardíaco que apresentavam sinais vitais à admissão na sala de operações. Método: Realizou-se um estudo descritivo e analítico, de cunho retrospectivo, através dos prontuários de 52 pacientes, submetidos à toracotomia para tratamento de lesões cardíacas penetrantes entre julho/91 e março/97 em um centro de trauma no Nordeste do Brasil. Resultados: $\mathrm{O}$ instrumento lesivo mais freqüente foi a arma branca em 28 pacientes $(53,85 \%)$, seguidos pela arma de fogo em 24 (46,15\%). A lesão de ventrículo direito isolada ocorreu em 25 casos $(48,08 \%)$. As medianas dos escores anatômicos de trauma foram: injury severity score $($ ISS $)=26$; penetrating trauma index $(\mathrm{PTI})=23$; penetrating thoracic trauma index $(\mathrm{PTTI})=20$; e penetrating cardiac trauma index $(\mathrm{PCTI})=15$. A taxa de complicação pós-operatória foi de $36,54 \%$ e a de letalidade de $13,45 \%$. O sangramento pós-operatório, a necessidade de reoperação e o uso de mais de três concentrados de hemácias aumentaram o risco relativo de morte. Os escores anatômicos de trauma não estiveram associados a aumento do risco relativo de letalidade. Conclusões: A maioria dos pacientes com lesão cardíaca desta série apresentou bons fatores prognósticos, justificando a baixa taxa de mortalidade observada.
\end{abstract}

Descritores: Traumatismo cardíaco; Feridas penetrantes.

\section{INTRODUÇÃO}

O primeiro tratamento para uma lesão cardíaca traumática em humano foi realizado por Williams em 1893, que fez uma sutura pericárdica em um homem com uma ferida penetrante de coração por arma branca, com necessidade posterior de uma reoperação de urgência para uma drenagem pericárdica ${ }^{1}$. A primeira sutura cardíaca em humanos foi realizada por Rhen em $1897^{2}$. Depois disso, a literatura reporta um crescente número de pacientes com trauma cardíaco, com diferentes mecanismos de lesão. Além disto, novas tecnologias como a sutura mecânica do miocárdio com esforços para reduzir a chance de contaminação durante o procedimento também foram apresentados ${ }^{3}$.

Lesões penetrantes de coração têm constituído um desafio para as equipes de trauma devido à grande letalidade $\mathrm{e}$ à urgência de procedimentos rápidos e agressivos ${ }^{4-12}$.

Em países desenvolvidos, o crescimento da violência urbana e incremento da assistência pré-hospitalar são responsáveis por um aumento potencial no número de pa-

1. Cirurgião Geral da Emergência de Adultos do Hospital da Restauração (HR) e Mestre em Cirurgia da UFPE.

2. Residente de Cirurgia Geral do Hospital das Clínicas da UFPE.

3. Mestre em Cirurgia da UFPE.

Recebido em 6/10/1999

Aceito para publicação em 26/9/2000

Trabalho desenvolvido na Emergência de Adultos do Hospital da Restauração da Universidade Federal de Pernambuco (UFPE). 
cientes com lesão penetrante de coração que chegam com vida aos centros de trauma ${ }^{5,7,13-17}$.

O objetivo deste estudo é determinar a incidência de complicações pós-operatórias e a taxa de letalidade do trauma cardíaco numa população atendida no Hospital da Restauração, localizado no Nordeste do Brasil, e verificar os fatores de risco associados (mecanismo e sítio anatômico da lesão, local da intervenção cirúrgica, lesões associadas e escores anatômicos de trauma).

\section{MÉTODO}

O Hospital da Restauração é o centro de referência de trauma no estado, realizando uma média de 500 operações de emergência por mês entre todas as especialidades. Cerca de $80 \%$ delas são decorrentes de traumatismo.

A principal causa de morte violenta no Estado de Pernambuco são as feridas penetrantes produzidas compreendendo cerca de $50 \%$ delas (dados do Instituto Médico Legal de Pernambuco - IML ).

A população estudada foi composta por 52 pacientes admitidos na Emergência do Hospital da Restauração - Recife - Brasil durante o período de julho de 91 a março de 97 , operados devido a traumatismo penetrante de coração e seguidos durante um período médio de internação de 6,3 dias. A mediana da idade foi 26 anos com variação de sete a 46 anos. Quarenta e nove casos $(94,33 \%)$ eram do sexo masculino.

Os critérios de inclusão usados foram: (1) presença de lesão penetrante de miocárdio confirmada durante o ato cirúrgico; (2) presença de sinais vitais à admissão na sala de operação. Pacientes transferidos no período pós-operatório para outros hospitais foram excluídos.

Um paciente que sofreu ferida penetrante por queda sobre espinho de Cereus jamacaru, uma planta xerófita semelhante a um cactus, e outro paciente com lesão produzida por instrumento manufaturado puntiforme foram considerados portadores de ferimento por arma branca.

Os pacientes foram inicialmente classificados segundo o mecanismo de trauma, sinais clínicos à admissão, indicação cirúrgica, uso de concentrado de hemácias, incisões usadas, incidência de complicações pós-operatórias e taxa de letalidade.

O grupo foi analisado como uma coorte retrospectiva. Essa coorte foi estratificada em vários graus de exposição (critérios de gravidade) a fim de comparar a incidência de complicações pós-operatórias e taxa de letalidade.

$\mathrm{O}$ risco relativo $(\mathrm{RR})$ de morte para cada parâmetro com intervalos de confiança de $95 \%$ foi calculado. O teste exato de Fisher bicaudal foi usado para verificar a diferença estatística do risco relativo. Um valor de $\mathrm{p}<0,05$ foi considerado estatisticamente significativo.

\section{RESULTADOS}

\section{Estudo descritivo}

Nos pacientes estudados, o instrumento lesivo mais usado foi a arma branca $(53,85 \%)$. O quadro clínico à admissão mais comum foi o choque hipovolêmico $(80,77 \%)$. O critério de indicação cirúrgica mais freqüente foi o choque persistente $(67,31 \%)$. Trinta e oito pacientes $(73,08 \%)$ fizeram uso de concentrado de hemácias no período pré e transoperatório. A incisão mais usada foi a toracotomia anterolateral esquerda $(69,23 \%)$. Dezenove pacientes $(36,54 \%)$ apresentaram complicações pós-operatória e sete $(13,46 \%)$ morreram (Tabela 1$)$. Vinte e um pacientes $(40.38 \%)$ apresentaram lesão em outro segmento do corpo. $\mathrm{O}$ abdome foi o sítio mais comum (20 pacientes). Os órgãos mais comumente lesados foram o pulmão esquerdo e o fígado (Tabela 2). O ventrículo direito foi a câmara cardíaca mais lesada $(48,08 \%)$, seguida pelo ventrículo esquerdo $(34,62 \%)$. Seis pacientes apresentavam lesões de mais de uma estrutura no coração (Tabela 3). Os escores anatômicos de trauma dos pacientes estudados estão apresentados na Tabela 4. A complicação pósoperatória mais frequente foi o sangramento (cinco pacientes $-9,62 \%)$, seguida pelo distúrbio metabólico grave (quatro pacientes $-7,69 \%$ ) (Tabela 5). Sete pacientes $(13,46 \%)$ foram submetidos a oito reoperações. A causa

\section{Tabela 1}

Mecanismo de trauma, sinais à admissão na emergência, indicações de cirurgia, uso de concentrado de hemácias e incisões.

\begin{tabular}{l|l|l}
\hline Parâmetros & No & $\%$ \\
\hline
\end{tabular}

\section{Mecanismo de trauma}

Arma branca

Arma de fogo

Sinais vitais à admissão na emergência

Choque hipovolêmico

Tamponamento cardíaco

Parada cardiorrespiratória

\section{Indicações para operação}

Choque hipovolêmico persistente

Tamponamento cardíaco

Parada cardiorrespiratória

$\begin{array}{rrr}42 & 80,77 \\ 9 & 17,31 \\ 1 & 1,92\end{array}$

Uso de concentrados de hemácias

Sim

Não

73,08
$14 \quad 26,92$

Incisões usadas

Toracotomia anterolateral esquerda

Toracotomia anterolateral bilateral

Esternotomia

Outras incisões

67,31

25,00

3,85

Complicações pós-operatórias

Não

Sim

63,46

Letalidade

Não

86,54

$\begin{array}{lll}\text { Sim } & 7 & 13,46\end{array}$ 
mais freqüente foi sangramento (quatro pacientes 7,90\%). Em três deles, era decorrente de vasos da parede torácica. Ao momento do procedimento, dois estavam em coma profundo e choque persistente e faleceram após a intervenção. O outro teve sua segunda lesão cardíaca descoberta e tratada na segunda reoperação. Esse segundo ferimento era puntiforme e localizado posteriormente no ventrículo esquerdo. Este recebeu alta hospitalar sem outras complicações ou seqüelas.

Tabela 2

Incidência de lesões associadas*

\begin{tabular}{l|r|r}
\hline Órgão & No & \multicolumn{1}{c}{$\%$} \\
\hline Órgãos torácicos & 21 & 40,38 \\
$\quad$ Pulmão esquerdo & 15 & 28,85 \\
Pulmão direito & 6 & 11,54 \\
Grandes vasos & 4 & 7,69 \\
Outros & 2 & 3,85 \\
Órgãos abdominais & 20 & 38,46 \\
Fígado & 13 & 25,00 \\
Estômago & 6 & 11,54 \\
Baço & 3 & 5,78 \\
Grandes vasos & 2 & 3,85 \\
Cólon & 2 & 3,85 \\
Rim esquerdo & 2 & 3,85 \\
Extremidades & 2 & 3,85 \\
Face e pescoço & 2 & 3,85 \\
\hline
\end{tabular}

*Havia pacientes com mais de uma estrutura lesada.

Tabela 3

Sítio da lesão cardíaca*

\begin{tabular}{l|r|r}
\hline Sítio da lesão & No & \multicolumn{1}{c}{$\%$} \\
\hline Ventrículo direito & 25 & 48,08 \\
Ventrículo esquerdo & 18 & 4,62 \\
Átrio direito & 9 & 17,31 \\
Vasos coronários & 3 & 5,80 \\
Átrio esquerdo & 2 & 3,85 \\
Septo interventricular & 2 & 3,85 \\
Valvas cardíacas & 1 & 1,92 \\
\hline
\end{tabular}

*Havia pacientes com mais de uma estrutura lesada.
Outras reoperações foram devidas à sepse abdominal (dois casos $-3,95 \%$ ) e tamponamento cardíaco. Num dos pacientes reoperados devido à sepse abdominal havia uma lesão despercebida e este faleceu durante a operação (Tabela 6). O quinto paciente a apresentar sangramento pós-operatório, estava estável na enfermaria quando apresentou sangramento abrupto, morrendo antes de chegar à sala de operação. Entre os cinco pacientes que apresentaram sangramento pós-operatório, três morreram (60\%).

Tabela 4

Escores anatômicos de trauma

\begin{tabular}{l|c|c|c}
\hline Escore & $\begin{array}{c}\text { Min. } \\
\text { valor }\end{array}$ & Mediana & $\begin{array}{l}\text { Max. } \\
\text { valor }\end{array}$ \\
\hline Injury Severity Score & 26 & 26 & 55 \\
Penetrating Trauma Index & 5 & 23 & 58 \\
Penetrating Thoracic Trauma & 5 & 20 & 38 \\
$\quad$ Index & & & \\
$\quad \begin{array}{l}\text { Penetrating Cardiac Trauma } \\
\quad \text { Index }\end{array}$ & 5 & 15 & 25 \\
\end{tabular}

Tabela 5

Complicações pós-operatórias

\begin{tabular}{l|c|c}
\hline Complicações & No & $\%$ \\
\hline Sangramento & 5 & 9,62 \\
Distúrbio metabólico grave & 4 & 7,69 \\
Arritmias & 3 & 5,77 \\
Pericardite & 2 & 3,85 \\
Sepse abdominal & 2 & 3,85 \\
Infecção de ferida & 2 & 3,85 \\
Infecção respiratória & 1 & 1,92 \\
Tamponamento cardíaco & 1 & 1,92 \\
Isquemia miocárdica & 1 & 1,92 \\
Fístula broncopleural & 1 & 1,92 \\
Defeito septo interventricular & 1 & 1,92 \\
Insuficiência aórtica & 1 & 1,92
\end{tabular}

Tabela 6

Causas de reoperação

\begin{tabular}{|c|c|c|c|c|c|c|}
\hline \multirow[b]{3}{*}{ Causas } & \multicolumn{4}{|c|}{ Morte } & & \\
\hline & \multicolumn{2}{|c|}{ Sim } & \multicolumn{2}{|c|}{ Não } & \multicolumn{2}{|c|}{ Total } \\
\hline & $n$ & $\%$ & $n$ & $\%$ & $n$ & $\%$ \\
\hline Sangramento & 2 & 28,57 & 2 & 28,57 & 4 & 57,14 \\
\hline Sepse abdominal & 1 & 14,29 & 1 & 14,29 & 2 & 28,57 \\
\hline Tamponamento cardíaco & - & - & 1 & 14,29 & 1 & 14,29 \\
\hline Total & 3 & 42,86 & 4 & 57,14 & 7 & 100,00 \\
\hline
\end{tabular}


Os outros três óbitos foram devidos a choque persistente e distúrbio metabólico grave no pós-operatório (Tabela 7). Cinco pacientes morreram nas primeiras $48 \mathrm{~h}$ de pós-operatório. Os outros no terceiro e quarto dias de após a operação.

Tabela 7

Causas de morte

\begin{tabular}{|c|c|c|}
\hline Causas & $N^{o}$ & $\%$ \\
\hline Sangramento pós-operatório & 3 & 42,86 \\
\hline $\begin{array}{l}\text { Choque grave e distúrbio } \\
\text { metabólico persistente }\end{array}$ & 3 & 42,86 \\
\hline Sepse abdominal & 1 & 14,28 \\
\hline Total & 7 & 100,00 \\
\hline
\end{tabular}

Dois pacientes foram submetidos à toracotomia em sala de emergência. Ambos faleceram.

No grupo estudado, a necessidade de transfusão de mais de três unidades de concentrado de hemácias $(\mathrm{RR}=8,18)$, sangramento pós-operatório $(\mathrm{RR}=7,06)$ e necessidade de reoperação $(R R=4,82)$ apresentaram-se como fatores de risco para letalidade $(\mathrm{p}<0,05)$.

O tipo de arma utilizada, a presença de lesão abdominal associada, o sítio da lesão cardíaca e os escores anatômicos de trauma não produziram elevações estatisticamente significativas no risco relativo $(\mathrm{p}>0,05)$ (Tabela 8).

\section{DISCUSSÃO}

Com o aumento da violência urbana, a literatura vem mostrando uma grande incidência de lesões cardiovasculares e em tórax ${ }^{15}$. Em Pernambuco, $9 \%$ das mortes violentas ocorridas em 1991 apresentaram ferimentos no coração ${ }^{18}$. A violência estende-se a crianças e adolescentes em países desenvolvidos ${ }^{19,20}$ e em nosso meio, com a observação de baixa média etária e de criança de sete anos no estudo ${ }^{21}$.

A arma branca foi o agente lesivo predominante. $\mathrm{O}$ traumatismo cardíaco por arma branca é tido como menos grave que o produzido por projétil de arma de fogo ou contusão, sendo apontado em diversos estudos como fator de bom prognóstico 6, 7,13,22,23. Uma vez que foram estudados apenas pacientes que chegaram com sinais vitais ao centro cirúrgico, é provável que os com menor gravidade tenham sido selecionados. Esta hipótese é corroborada por estudo anterior em falecidos por morte violenta no Estado no ano de 1991 , onde $70 \%$ dos óbitos com trauma cardíaco foram produzidos por arma de fogo ${ }^{18}$.

Estima-se que cerca de $80 \%$ dos pacientes com traumatismo cardíaco não recebam assistência médica 7, 8, 22 . Em Pernambuco, apenas $6 \%$ dos que falecem devido a trauma cardíaco recebem algum tipo de assistência ${ }^{18}$. A predominância do choque hipovolêmico à admissão e como principal critério de indicação cirúrgica converge com a literatura e denota a gravidade deste grupo.
A melhora dos serviços de medicina pré-hospitalar, com assistência adequada e rápida mobilização torna possível a chegada de pacientes com melhores condições fisiológicas aos centros de trauma em países desenvolvidos 7, 24. Durham III et al., observaram que traumatizados em parada cardiorrespiratória (PCR) que chegavam à sala de emergência intubados e em ventilação artificial apresentavam o dobro do tempo de sobrevida dos não intubados ${ }^{25}$.

No Recife, o atendimento pré-hospitalar é coordenado pelo Corpo de Bombeiros, sendo composto também por médicos. Este serviço começou em 1995 e nos seis primeiros meses havia trazido aos centros de trauma menos de $10 \%$ dos pacientes traumatizados ${ }^{26}$. Não houve aumento da sua capacidade, estimando-se que atenda atualmente um número similar de pacientes.

Dois pacientes foram submetidos à toracotomia ressuscitativa em sala de emergência (TRSE) devido à parada cardiorrespiratória durante o atendimento. Esse procedimento é acompanhado de elevada mortalidade, que varia entre 73 e $100 \%$ 14, 23, 24, 27 - 29. Devido ao seu alto custo, diversos protocolos foram propostos para seleção de pacientes. Vítimas de trauma torácico penetrante que chegam com sinais vitais em sala de emergência têm o melhor prognóstico ${ }^{8,10,11}$, sendo este o critério adotado no serviço. A importância da TRSE no trauma contuso é questionada devido aos pobres resultados em temos de sobrevida, com esta variando entre 0 e $6 \%$ 22, 27, 28 . Pacientes traumatizados que chegam em assistolia apresentam mortalidade relatada de $100 \%$ 6, 25 . Nos dois casos descritos no estudo em que obteve-se sucesso na ressuscitação em sala de emergência os pacientes faleceram após a operação definitiva.

Como esperado devido à gravidade da população estudada, a grande maioria necessitou de transfusão de hemoderivados durante o período pré e transoperatório.

A incisão mais freqüentemente usada foi a toracotomia anterolateral esquerda. Esta incisão demonstra-se adequada quanto à exposição de praticamente todo o coração, tornando possível sua realização, pericardiotomia e abordagem do coração de forma extremamente rápida, sendo indicada nos casos de instabilidade hemodinâmica ${ }^{11}$, como a maior parte de nossa população. Quando da presença de lesões em átrio direito ou vasos da base por vezes faz-se necessária a extensão da incisão para o tórax direito, procedimento que é realizado também de forma rápida e sem acréscimo significativo de morbidade. A esternotomia não é incisão preferencial neste serviço uma vez que aumenta a demanda de tempo e os riscos de osteomielite devido ao procedimento ser realizado em condições de urgência, muitas vezes com lesão de víscera oca abdominal associada.

Observou-se um índice de complicações pós-operatórias de $36,54 \%$. Este índice é similar a outro levantamento local onde estudaram-se as todas as toracotomias devidas a trauma ${ }^{30}$. Na literatura mundial, a consideração sobre morbidade é muitas vezes colocada em segundo plano em relação à mortalidade, sendo diversos os critérios para o enquadramento destas ${ }^{23}$. Num estudo retrospectivo, estas complicações ficam subdimensionadas. Praticamente todo paciente que apresenta 
Tabela 8

Risco relativo de morte associado com o mecanismo de trauma, uso de concentrado de hemácias, lesão abdominal associada, sítio de lesão cardíaca, sangramento pós-operatório, necessidade de reoperação e escores de trauma

\begin{tabular}{|c|c|c|c|c|c|}
\hline Parâmetro & Morte & Sobrevida & $R R$ & I.C. $p / 95 \%$ & $P$ \\
\hline \multicolumn{6}{|l|}{ Mecanismo de trauma } \\
\hline Arma de fogo & 2 & 22 & 0,42 & $0,10-\quad 2,19$ & 0,43 \\
\hline Arma branca & 5 & 23 & 1,00 & & \\
\hline \multicolumn{6}{|l|}{ Concentrado de hemácias } \\
\hline 4 ou mais unidades & 6 & 16 & 8,18 & $1,06-63,19$ & 0,03 \\
\hline 0-3 unidades & 1 & 29 & 1,00 & & \\
\hline \multicolumn{6}{|l|}{ Lesão abdominal associada } \\
\hline Sim & 3 & 17 & 1,20 & $0,30-\quad 4,81$ & 1,00 \\
\hline Não & 4 & 28 & 1,00 & & \\
\hline \multicolumn{6}{|l|}{ Sítio da lesão cardíaca } \\
\hline Mais de uma estrutura & 1 & 5 & 3,67 & $0,27-50,40$ & 0,38 \\
\hline Átrio esquerdo & 1 & 1 & 11,00 & $1,03-116,95$ & 0,16 \\
\hline Átrio direito & 2 & 7 & 4,89 & $0,50-47,41$ & 0,19 \\
\hline Ventrículo esquerdo & 2 & 16 & 2,44 & $0,24-24,83$ & 0,57 \\
\hline Ventrículo direito & 2 & 25 & 1,63 & $0,16-16,82$ & 1,00 \\
\hline Isolada de ventrículo direito & 1 & 21 & 1,00 & & \\
\hline \multicolumn{6}{|l|}{ Sangramento pós-operatório } \\
\hline Sim & 3 & 2 & 7,05 & $2,17-22,93$ & 0,01 \\
\hline Não & 4 & 43 & 1,00 & & \\
\hline \multicolumn{6}{|l|}{ Necessidade de reoperação } \\
\hline Sim & 3 & 4 & 4,82 & $1,36-17,13$ & 0,04 \\
\hline Não & 4 & 41 & 1,00 & & \\
\hline \multicolumn{6}{|l|}{ Injury severity score } \\
\hline $35-51$ & 3 & 11 & 2,07 & $0,48-\quad 8,99$ & 0,37 \\
\hline $27-34$ & 1 & 8 & 1,07 & $0,13-\quad 9,09$ & 1,00 \\
\hline 26 & 3 & 26 & 1,00 & & \\
\hline \multicolumn{6}{|c|}{ Penetrating thoracic trauma index } \\
\hline $25-38$ & 2 & 10 & 1,28 & $0,25-\quad 6,64$ & 1,00 \\
\hline $16-24$ & 2 & 15 & 0,90 & $0,17-\quad 4,82$ & 1,00 \\
\hline $5-15$ & 3 & 20 & 1,00 & & \\
\hline \multicolumn{6}{|l|}{ Penetrating trauma index } \\
\hline $29-58$ & 3 & 10 & 2,65 & $0,51-13,89$ & 0,32 \\
\hline $21-28$ & 2 & 14 & 1,44 & $0,23-\quad 9,17$ & 1,00 \\
\hline $5-20$ & 2 & 21 & 1,00 & & \\
\hline \multicolumn{6}{|c|}{ Penetrating cardiac trauma index } \\
\hline $20-25$ & 3 & 17 & 0,90 & $0,21-\quad 3,91$ & 1,00 \\
\hline $11-19$ & 1 & 13 & 0,43 & $0,05-\quad 3,69$ & 0,61 \\
\hline $5-10$ & 3 & 15 & 1,00 & & \\
\hline
\end{tabular}

choque hipovolêmico apresentará certo grau de acidose metabólica num momento posterior. Neste estudo foram classificados como portadores de distúrbio metabólico grave os pacientes em que havia registro das gasimetrias e tentativa de correção medicamentosa das mesmas. É interessante observar que a baixa taxa de infecção de ferida é a norma, como observado também neste estudo, a despeito da urgência dos casos por vezes limitar a antisepsia adequada $11,30,31$.
Cha e Symbas relataram um número significativo de sequielas tardias devido a defeitos estruturais no coração (23 e $21 \%$, respectivamente). Na maior parte das vezes, este é suspeito durante a operação e o diagnóstico é confirmado até uma semana após o trauma com o aparecimento dos sinais clínicos. As seqüelas mais comuns são os defeitos septais ventriculares, defeitos valvares e distúrbios de condução ${ }^{32,33}$. Foram anotados nesta série, seis pacientes com lesão de mais de uma estrutura sendo três deles lesão 
de câmara associada à de vaso coronário. Os outros resultaram em defeito no septo ventricular (dois casos) e defeito valvar (um caso).

As feridas cardíacas complexas ocorrem mais comumente quando o agente lesivo é o projétil de arma de fogo. $\mathrm{Na}$ urgência, é recomendado apenas o reparo da parede externa do coração e posterior avaliação da necessidade de correção de outras lesões, se a condição hemodinâmica do paciente permitir ${ }^{32,33}$.

A baixa letalidade observada não pode ser comparada de imediato com outros estudos uma vez que possui critérios de inclusão diversos, admitindo só aqueles que chegam com sinais vitais ao centro cirúrgico. Este método aglutina pacientes que passaram por ampla seleção natural e reúnem boas condições para o trabalho do cirurgião. Observa-se na literatura uma grande variação quanto as taxas de mortalidade nos diferentes estudos, devido a diferenças das características locais dos traumas (mecanismo de lesão, eficácia do atendimento pré-hospitalar etc.) e aos critérios de inclusão utilizados, motivos estes que levaram à concepção de normatização que tornasse possível a comparação das populações (escores de trauma) ${ }^{7,14,16}$.

Encontrou se neste trabalho uma tendência à diminuição do risco relativo (RR) em pacientes com lesão por arma de fogo, porém de forma não significativa estatisticamente. Acreditamos que estes casos sejam fruto de feridas produzidas por arma de menor calibre, uma vez que sabemos do maior poder letal desta lesão $6,7,13,22,23$.

Os pacientes que necessitaram de mais de três concentrados de hemácias nos períodos pré e transoperatório apresentaram um maior RR para a morte $(\mathrm{p}<0,05)$, o que denota a gravidade do grupo. Nenhum dos que não necessitaram de transfusão faleceu.

A presença de lesão abdominal associada não aumentou de forma estatisticamente significativa o RR. Quando observamos o estudo de Carvalho et al. ${ }^{18}$ entre pacientes falecidos com lesão cardíaca no Estado, encontramos lesões associadas em $90 \%$ dos casos. Apesar de se saber que algumas que acometem o coração são incompatíveis com a vida, a presença das lesões associadas certamente influencia na letalidade, embora não se tenha caracterizado o fato neste estudo. É provável que a forma de seleção e pequeno número de casos seja responsável pelo resultado.

Vinte e dois pacientes apresentaram lesão única de ventrículo direito. Esta lesão é produzida usualmente por arma branca, uma vez que esta câmara apresenta-se na face anterior do tórax, próxima à parede, sendo suscetível aos ataques frontais com estes instrumentos que apresentam baixo poder de penetração. Esta é considerada a lesão cardíaca mais fácil de lidar, uma vez que não há comprometimento de lesão tecidual e a própria musculatura ventricular poderá tamponá-la parcialmente além do fácil acesso para o cirurgião $4,7,13,15,16,23,34,35$. As lesões atriais são consideradas de maior gravidade pelo sangramento contínuo que apresentam por não haver possibilidade de tamponamento pela sua fina parede. Neste estudo, a elevação do RR para as lesões de átrio direito e principalmente átrio esquerdo (RR de 4,89 e 11,00 respectivamente) não apresentaram significado estatístico $(\mathrm{p}>0,05)$. Atribuiu-se ao baixo número de casos de pacientes estudados com estas lesões.

Sangramento pós-operatório e a necessidade de reoperação são eventos interligados. Neste estudo, ambos causaram aumento do RR para a morte $(\mathrm{p}<0,05)$. O sangramento foi responsável por quatro das oito reoperações.

Hishberg demonstrou $2 \%$ de reoperações por sangramento após operação por trauma. O tórax foi o segundo sítio de sangramento sendo as lesões de vasos da parede a primeira causa de sangramento torácico ${ }^{36}$. Possivelmente devido ao choque hipovolêmico o sangramento não esteja ativo ao momento da síntese torácica, retornando a sangrar após a estabilidade hemodinâmica. O mesmo autor observou seis lesões cardíacas despercebidas em 49 reoperações no tórax ${ }^{36}$. Houve neste levantamento um caso de lesão cardíaca apenas percebida na segunda reoperação.

A necessidade de aprofundar o estudo do trauma trouxe a exigência de uma melhor classificação dos pacientes. Foram formulados os escores de trauma divididos em anatômicos e fisiológicos ${ }^{4,14,17}$. Devido a características próprias, os escores fisiológicos relacionam-se com a mortalidade nas primeiras $24 \mathrm{~h}$ e os escores anatômicos com a mortalidade tardia. Em grupos de pacientes com instabilidade hemodinâmica, como o estudado, os escores fisiológicos seriam úteis. Não foi possível a coleta retrospectiva de dados acerca do estado funcional dos pacientes (tensão arterial, freqüência cardíaca e respiratória etc.).

Os escores anatômicos de trauma utilizados apresentaram comportamento particular. Quando observa-se o Injury Severity Score (ISS) e Penetrating Trauma Index (PTI), que consideram as lesões encontradas em todo o corpo e o Penetrating Thoracic Trauma Index (PTTI), que leva em conta a soma das lesões torácicas, nota-se um gradiente de aumento do RR para os maiores valores de escore, a despeito de não apresentarem diferença estatística significativa ( $\mathrm{p}>0,05)$, de forma similar à que se observou nas lesões abdominais associadas. É possível que o pequeno número de pacientes estudados tenha influenciado. O Penetrating Cardiac Trauma Index (PCTI), que leva em conta apenas características da lesão cardíaca não apresentou relação com a gravidade.

Em conclusão, os pacientes portadores de traumatismo cardíaco penetrante, admitidos com sinais vitais no bloco cirúrgico e operados tem características próprias, com predomínio das lesões isoladas, por arma branca, e em ventrículo direito, fatores de bom prognóstico, o que determinou a baixa letalidade do grupo estudado. Pacientes que utilizaram mais de três unidades de concentrado de hemácias no período pré e transoperatório e que apresentaram no pós-operatório sangramento ou necessidade de reoperação tiveram um aumento no RR para a morte. $\mathrm{O}$ escores anatômicos de trauma não apresentaram relação estatisticamente significativa com letalidade. 


\begin{abstract}
Background: The purpose of this study is to outline the postoperative complications, lethality rates and associated risk factors (trauma mechanism, anatomic site injury, location of surgical intervention, associated injuries, transfusion need and anatomic trauma scores) in patients with cardiac wounds presenting vital signs at admission in the operation room. Methods: This is a retrospective and analytic study using charts of 52 patients, who underwent thoracotomy for treatment of cardiac injuries in a trauma center in northeastern Brazil between July/ 91 and March/97. Results: The most frequent injury was caused by stab wound, in 28 patients (53.85\%), followed by gunshot wounds in 24 patients (46.15\%) Isolate right ventricle wounds occurred in 25 cases (48.08\%). The median for anatomic trauma scores were: injury severity score $(I S S)=26$; penetrating trauma index $(P T I)=23$; penetrating thoracic trauma index $(P T T I)=20$; and penetrating cardiac trauma index $(P C T I)=15$. Postoperative complication rate was $36.54 \%$ and the lethality rate was $13.45 \%$. Postoperative bleeding, reoperation and use of more than three packaged red blood cells increased de relative risk to lethality. The anatomic trauma scores were not associated with the relative risk to lethality. Conclusion: The majority of patients treated for cardiac injuries presented good predictors for survival, justifying our low mortality rates.
\end{abstract}

Key Words: Cardiac trauma; Penetrating wounds.

\title{
REFERÊNCIAS
}

1. Williams DH.- Stab wound of the heart and the pericardium - suture of the heart - recovery - patient alive three years afterward. Med. Rec. 1897;437.

2. Rhen L. - Ubber penetirende. Herwunden und Herznaht. Arch. Klin. Chir. 1897;55:315.

3. Macho JR, Markinson RE, Schecter WP. - Cardiac stapling in the management of penetrating injuries of the heart: rapid control and decreased risk of personal contamination. J. Trauma. 1993;34(5):711-5.

4. Arreola-Risa C, Rhee P, Boyle EM, et al. - Factors influencing outcome in stab wounds of the heart. Am. J. Surg. 1995; 169:553-6.

5. Baker S, O’Neill B, Haddon W, et al. - The injuty severity score: a method for describing patients with multiple injuries and evaluating emergency care. J. Trauma 1974;14(3):187-96.

6. Buchmann TG, Phillips J, Menker JB. - Recognition, resuscitation and management of patients with penetrating cardiac injuries. Surg. Gynecol Obstet. 1992;174:20510.

7. Coimbra R, Pinto MCC, Razuk A, et al. - Penetrating cardiac wounds: predictive value of trauma indices and the necessity or terminology standardization. Am. Surg. 1995;61(5):448-52.

8. Henderson VJ, Smith RS, Fry WR, et al. Cardiac injuries: analysis of an unselected series of 251 cases. J. Trauma 1994;36(3):341-8.

9. Kaplan AJ, Norcross D, Crawford FA. - Predictors of mortality in penetrating cardiac injury. Am. Surg. 1993;59:338-41.

10. Kulshrestha P, Iyer KS, Balram A, et al . - Chest injuries: a clinical and autopsy profile. J. Trauma 1988;28(6):844-7.

11. Mitchell ME, Muakkassa FF, Poole GV, et al. - Surgical aproach of choice for penetrating cardiac wounds. J. Trauma 1993;34(1):17-20.

12. Velmahos GC, Degiannis E, Souter I. - Outcome of a strict policy on emergency department thoracotomies. Arch. Surg. 1995;130:774-7.
13. Baccarin V, Rizoli SB, Vieira RW, et al.- Ferimentos penetrantes cardíacos - experiência com 21 casos. Rev. Col. Bras. Cir. 1992;19(3):112-5.

14. Ivatury RR, Nallathambi MN, Stahl WM. - Penetrating cardiac trauma: quantifying the severity of anatomic and physiologic injury. Ann. Surg. 1987;205:61-6.

15. Mattox KL, Feliciano DV, Burch J, et al. - Five thousand seven hundred sixty cardiovascular injuries in 4459 patients. Ann. Surg. 1989;209(6):698-707.

16. Moore EE, Malangoni MA, Cogbill TH, et al. - Organ injury scaling IV: thoracic vascular, lung cardiac and diaphragm. J. Trauma. 1994;36:299-300.

17. Osler T. - Injury severity scoring: perspectives in development and future directions. Am. J. Surg. 1993;16S(2A):43S-51S.

18. Carvalho G, Cavalcanti AG, Spencer Netto FAC. - Trauma cardíaco: análise de 427 casos de necrópsia. Revista do Hospital da Restauração. 1993;4(1):23-6.

19. Laraque D, Barlow B, Durkin $M$ et al. - Children who are shot: a 30-year experience. J. Pediatr. Surg. 1995;30:1072-5.

20. Rheinhorn M, Kaufman HL, Hirsch EF, et al. - Penetrating thoracic trauma in a pediatric population. Ann. Thorac. Surg. 1996;61:1501-5.

21. Spencer Netto FAC, Melo PSV, Santos Filho OR. - Traumatismo cardíaco com retenção de corpo estranho em criança de sete anos. Rev. Col. Bras. de Cir 1998;25:285-6.

22. Moreno C, Moore E, Mjure JA, et al. - Pericardial tamponade: a critical determinant for survival following penetrating cardiac wounds. J. Trauma 1986;26:821-5.

23. Velmahos GC, Degiannis E, Souter I, et al. - Penetrating trauma to the heart: a relatively innocent injury. Surgery 1994;115:694-7.

24. Blake DP, Gisbert VL, Ney AL, et al. - Survival after emergency department versus operating room thoracotomy for penetrating cardiac injuries. Am. Surg. 1992;58:329-33. 
25. Durham III LA, Richardson RJ, Wall MJ, et al. - Emergency center thoracotomy: impact of prehospital resuscitation. J. Trauma 1992;32:775-9.

26. Melo IS, Spencer Netto FAC, Arnold MW, et al. - Avaliação inicial do impacto do serviço de atendimento prehospitalar na Grande Recife: atuação do corpo de bombeiros. Panamerican J. Trauma 1995;5(2):75.

27. Boyd M, Vanek VW, Bourguet CC. Emergency room resuscitative thoracotomy: when is it indicated ? J. Trauma 1992;33:714-21.

28. Kavalius J, Golocovsky M, Champion HR. - Predictors of outcome in patientes who have sustained trauma and undergo emergency thoracotomy. Arch. Surg. 1993; 128:1158-62.

29. Mazzorana V, Smith RS, Morabito DJ, et al. - Limited utility of emergency department thoracotomy. Am. Surg. 1994;60:516-20.

30. Spencer Netto FAC, Lima LFC, Campos JM, et al. - Toracotomias por trauma: análise das complicações e mortalidade tardia. An. Fac. Med. Univ. Fed. Pernamb. 1996;41:124-30.

31. Demetriades D. - Cardiac wound: experience with 70 patients. Ann. Surg. 1986;203:315-7.

32. Cha EK, Mittal V, Allaben RD. - Delayed sequelae of penetrating cardiac injury. Arch. Surg. 1993;128:836-9.

33. Symbas PN, DiOrio DA, Tyras DH, et al. - Penetrating cardiac wounds: significant residual and delayed sequelae. J. Thorac. Cardiovasc. Surg. 1973; 66:526-32.
34. Buchmann Jr RF, Badelino MM, Mauro LH, et al. - Penetrating cardiac wounds: prospective study factors influencing initial resuscitation. J. Trauma 1993;34:71727.

35. Naughton MJ, Brissie RM, Bessey PQ, et al. - Demography of penetrating cardiac trauma. Ann. Surg. 1989;209:676-83.

36. Hirshberg A, Wall MJ, Ramchandani MK, et al. - Reoperation for bleeding in trauma. Arch. Surg. 1993;128:1163-7.

Endereço para correspondência:

Dr. Fernando Antônio Spencer Netto

Rua Fernando Simões Barbosa, 50/102

51020-390 — Recife-PE

E-mail: kummer@netpe.com.br 